\title{
Anestesia espinal parte IV. Técnica de la anestesia espinal y sus variaciones
}

\section{Spinal anesthesia part IV. Spinal anesthesia technique and its variations}

Fernando Altermatt C. ${ }^{1}$, Juan Carlos De La Cuadra F. ${ }^{1}$, Catalina Kychenthal L. ${ }^{2}$, María Jesús Irarrázaval M.², Héctor Lacassie Q. ${ }^{1}$

\begin{abstract}
Spinal anesthesia is a very important and widely used technique nowadays. In order to obtain successful results and avoid complications, it is essential to perform it with the correct methods. The operator must be familiar with the procedure and prepare correctly for it, including material and drug selections which may vary according to the patient. Risk reduction is fundamental, and it is accomplished by carrying out the previously mentioned preparation and an adequate monitorization of the patient being intervened. Once this is in order, the patient must be positioned into being sited, or placed in supine or lateral position. The chosen approach depends mainly on the characteristics of anatomical repairs and may be medial, paramedian or a Taylor approach. The correct needle must be selected, and these are categorized mainly into those which cut or separate the dura's fibers. In this revision, the correct technique for performing spinal anesthesia is described, along with some of its most important variations such as sectorized anesthesia, continuous spinal anesthesia and combined epidural spinal anesthesia.
\end{abstract}

\section{RESUMEN}

La anestesia espinal es una técnica ampliamente utilizada hoy en día. Para obtener resultados exitosos y evitar complicaciones, es fundamental una buena técnica a la hora de realizarla. Debe contarse con un buen conocimiento basal

\section{Key words:}

Anesthesia,

regional anesthesia, spinal anesthesia, local anesthesia

\section{Palabras clave:}

Anestesia, anestesia regional, anestesia espinal, anestésico local

División de Anestesiología, Escuela de Medicina, Pontificia Universidad Católica de Chile. Santiago, Chile.

Escuela de Medicina, Pontificia Universidad Católica de Chile. Santiago, Chile.

Fecha de ingreso: 22 de octubre de 2020

Fecha de aceptación: 11 de noviembre de 2020

\section{ORCID}

0000-0002-0464-8643

Sin fuentes de financiamiento.

No se declaran conflictos de interés.

Correspondencia:

Fernando Altermatt Couratier

falterma@uc.cl 
del método a utilizar y una preparación adecuada según el paciente, incluyendo en esto tanto los materiales como los fármacos de elección en cada caso. Es fundamental reducir los riesgos tanto locales como sistémicos del procedimiento, lo cual se logra con la preparación mencionada y una adecuada monitorización del paciente. Una vez listo esto, debe posicionarse al paciente de manera que quede sentado, en decúbito lateral o decúbito prono. El abordaje es elegido principalmente según los reparos anatómicos de cada caso particular y puede ser medio, paramediano o de Taylor. Se debe seleccionar una aguja adecuada, las cuales se dividen principalmente entre aquellas que cortan y aquellas que separan las fibras de la duramadre. En esta revisión se expone la técnica adecuada para realizar el procedimiento de anestesia espinal, junto con algunas de sus variaciones principales como lo son la anestesia sectorizada, la anestesia espinal continua y la anestesia combinada espinal epidural.

\section{Introducción}

$\mathrm{R}$ ealizar un procedimiento de anestesia regional con éxito requiere el seguir meticulosamente una serie de pasos, que comienzan con la adecuada preparación del paciente y el instrumental a usar, pasan por la inspección y análisis de los reparos anatómicos del paciente y terminan en una técnica prolija.

La preparación considera el equipo y la elección de la o las drogas a utilizar, dependiendo de variables como las características del paciente: edad, parámetros biométricos, condición fisiológica, del tipo de cirugía, para determinar el nivel anestésico que se busca, y la duración de ésta.

Antes de la punción, se deben tomar todas las precauciones necesarias para disminuir los riesgos. Todo paciente debe ser monitorizado previo a cualquier maniobra, con al menos electrocardiograma continuo, oximetría de pulso y presión arterial no invasiva, así como contar con una vía venosa permeable por la cual poder administrar sedación o tratar potenciales complicaciones durante el procedimiento.

\section{Técnica tradicional}

\section{Posición del paciente}

La posición del paciente es probablemente uno de los determinantes más importantes de una anestesia espinal exitosa, pero es comúnmente pasado por alto.

Existen tres formas de posicionar pacientes para una punción espinal: sentado, decúbito lateral y decúbito prono. De ellas, las dos primeras son las más frecuentemente usadas.

El decúbito lateral permite una sedación más pro- funda, con un paciente más confortable aun cuando esté más sedado, requiriendo menos ayuda del asistente que colabora con la posición.

Los pacientes son puestos con su espalda paralela y al borde de la mesa de procedimientos, en posición fetal, arqueando la espalda, mientras se mantiene el cuello y las caderas flexionadas (Figura 1).

La posición sentada requiere más ayuda por parte del asistente, manteniendo al paciente en una posición más vertical, de modo de facilitar la identificación de la línea media, mientras se flexiona el cuello y arquea la espalda (Figura 2). Es útil el uso de una almohada en el regazo del paciente, que permite apoyar los codos en ella mientras se acentúa el arco de la espalda. Otro elemento útil es el uso de una banqueta para apoyar los pies del paciente, facilitando la flexión de las caderas.

El decúbito prono, si bien no es comúnmente usado en procedimientos anestésicos, y puede ser más frecuente para procedimientos intervencionales en dolor, resulta útil en pacientes sometidos a procedimientos anorrectales en posición de navaja suiza,

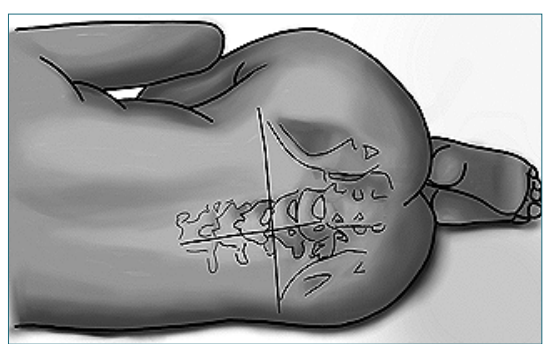

Figura 1. Posición en decúbito lateral para una punción espinal. La espalda paralela y al borde de la mesa de procedimientos, en posición fetal, arqueando la espalda, mientras se mantiene el cuello y las caderas flexionadas. Se muestran la línea media y la línea inter-crestal. 


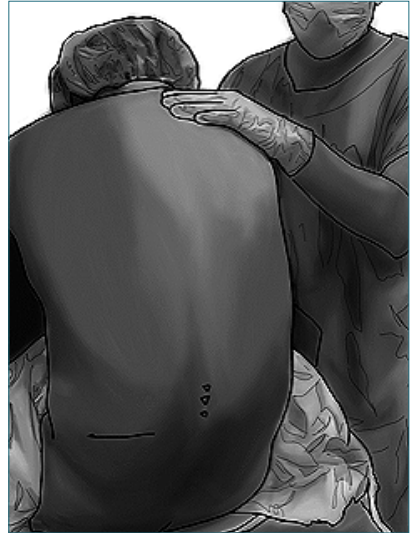

Figura 2. Posición en sentada para una punción espinal. Requiere más ayuda por parte del asistente, manteniendo al paciente en una posición más vertical, de modo de facilitar la identificación de la línea media, mientras se flexiona el cuello y arquea la espalda. Se muestran las apófisis espinosas y la línea inter-crestal.

usando drogas hipobáricas. Técnicamente resulta más difícil, puesto que en prono la tendencia natural de la columna lumbar es de mantener o incluso exacerbar su lordosis, dificultando el acceso por la línea media entre las apófisis espinosas, por lo que frecuentemente un abordaje paramediano puede resultar mejor. Asimismo, la salida del LCR puede estar limitada, por lo que el reflujo resulta más lento o inexistente.

La punción en sí misma exige una técnica aséptica rigurosa, dado que una de las complicaciones temidas de esta técnica lo constituyen las complicaciones infecciosas. Del mismo modo, en la preparación de las drogas a inyectar es crítica la identificación y doble chequeo de ellas, de modo de evitar errores de administración, con catastróficas consecuencias.

\section{Abordajes}

Existen dos formas de acceder al espacio subaracnoideo, independientemente de la posición del paciente: a través de un abordaje medio (el más comúnmente usado) y el paramediano.

\section{Abordaje medio}

El abordaje medio requiere una adecuada posición del paciente y una correcta identificación de los puntos de reparo anatómicos de superficie. A mayor flexión de la columna lumbar, mayor separación y apertura de los espacios interespinosos, facilitando la punción.

La clave radica en identificar la línea media, a través de la inspección y palpación de las apófisis espinosas lumbares bajas, frecuentemente de L3 a L5, y sus correspondientes interespacios (Figura 1).

En la práctica, la línea intercrestal o de Tuffier, una línea imaginaria uniendo la parte posterior de las crestas iliacas del paciente, comúnmente intercepta la apófisis espinosa de L4, por lo que se usa como referencia para identificar el nivel de la punción. Hay que tener en cuenta que la evidencia disponible demuestra que estos puntos de reparo tienen una amplia variación interindividual[1] y que en ocasiones subestima el nivel de punción, especialmente en pacientes obesos.

Tras una adecuada inspección, la palpación de las apófisis espinosas permite identificar la línea media y alteraciones rotacionales de la columna lumbar (por ejemplo, escoliosis), de modo de elegir el sitio más adecuado para la punción. Resulta conveniente usar el dedo índice y pulgar de la mano no dominante como una pinza agarrando la apófisis espinosa.

Se administra anestesia local en forma de una pápula en piel y tejido subcutáneo en el sitio elegido para la punción. Posteriormente, se punciona con el introductor, perpendicular a la piel en todos los planos, con una discreta inclinación de 10-15 grados cefálicos, hasta dejar éste fijo y anclado en el ligamento interespinoso. Es esencial conservar la orientación sobre la línea media. El introductor atravesará a su paso el tejido subcutáneo, el ligamento supraespinoso, y parte del ligamento interespinoso. Se debe ser cuidadoso, especialmente en pacientes muy delgados, de no introducir muy profundamente el introductor y perforar la duramadre con él.

Una vez fijo el introductor, se introduce lentamente el trócar espinal a través de éste, sintiendo su paso a través de los distintos planos del ligamento interespinoso, el ligamento amarillo, el espacio peridural, hasta vencer una resistencia leve, al atravesar la duramadre y aracnoides. Esta última resistencia es más evidente con trócares de punta no cortante o de mayor diámetro.

Al retirar el mandril, deberá observarse refluir LCR a través del trócar. Dependiendo del diámetro de la aguja espinal usada, el reflujo puede ser más o menos lento.

Posteriormente, se conecta la jeringa conteniendo la solución intratecal a administrar $\mathrm{y}$, tras asegurar la aspiración de LCR, se inyecta lentamente.

Es posible que aún habiendo atravesado los planos adecuados, no haya reflujo de LCR, especialmente en aquellas agujas con su portal lateral a la punta. En esos casos, una cuidadosa rotación en 90 grados de la aguja puede solucionar el problema. Otras veces, especialmente en agujas con diámetros más pequeños, la aspiración suave con una jeringa mientras se avanza, puede facilitar la identificación del espacio 
subaracnoideo[2].

La causa más común para no obtener una punción subaracnoidea exitosa, es perder la línea media durante la punción, que coincide muchas veces con el contacto con las láminas. Si en su trayecto, la aguja espinal contacta con hueso, debe ajustarse el sitio de punción, de modo de orientar el introductor y el trócar más a cefálico, para sortear la apófisis espinosa inmediatamente inferior.

\section{Abordaje paramediano}

En cierto tipo de pacientes, el abordaje a través de la línea media presenta dificultades que lo hacen impracticable. Pacientes ancianos, en los que los ligamentos interespinosos se encuentran calcificados, o aquellos con limitación a la flexión de la columna lumbar o lordosis muy pronunciadas, pueden tener una limitación al acceso al espacio subaracnoideo a través de la línea media.

Las ventajas del abordaje paramediano, adicionalmente, incluyen el que puede ser intentado independientemente de la posición del paciente o de su grado de flexibilidad de la columna lumbar.

Para realizarlo, es fundamental identificar la línea media y las apófisis espinosas. El sitio de punción esta vez será $1 \mathrm{~cm}$ lateral y $1 \mathrm{~cm}$ caudal a este punto, con una inclinación de 15 grados hacia medial, y 15 grados a cefálico. Estas referencias deben tener en cuenta que la angulación tanto en el eje parasagital como en el transverso deben dirigir nuestro introductor y aguja hacia el centro de la pared posterior del saco dural. Por tanto, debe entrenarse la percepción de la profundidad a la que éste se encuentra, y hacer correcciones en cuanto a distancias (caudal y lateral respecto a las apófisis espinosas) y en cuanto a ángulos (más agudos o más perpendiculares), dependiendo de

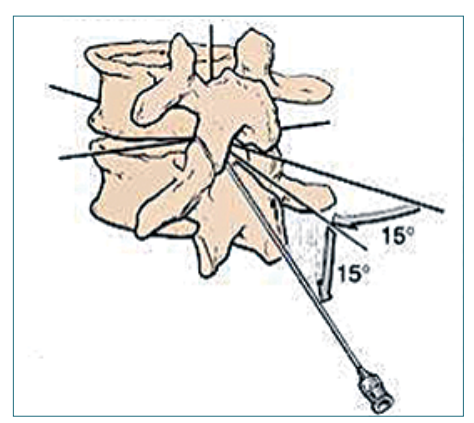

Figura 3. Abordaje paramediano para una punción espinal. Para realizarlo, es fundamental identificar la línea media y las apófisis espinosas. El sitio de punción esta vez será $1 \mathrm{~cm}$ lateral y $1 \mathrm{~cm}$ caudal a este punto, con una inclinación de 15 grados hacia medial y 15 grados a cefálico. la profundidad presunta del saco dural (Figura 3).

Por ser un trayecto oblicuo, la distancia recorrida es más larga y la aguja debe insertarse con mayor longitud de aguja. En el caso del abordaje paramediano, frecuentemente el plano de resistencia más evidente es el ligamento amarillo.

Por su dirección, es común que la aguja contacte hueso en este abordaje, normalmente la lámina, por lo que, en ese caso, se debe "cabalgar" hacia cefálico para acceder al espacio subaracnoideo.

\section{Abordaje de Taylor}

Estrictamente es un abordaje paramediano a nivel del interespacio entre L5 y S1[3]. Tiene la gran ventaja que aborda el espacio más amplio al que se puede acceder, por lo que es una buena opción cuando otros abordajes son impracticables o difíciles. Puede realizarse indistintamente en decúbito lateral, sentado o prono.

Los puntos de reparo en este caso lo constituyen la espina ilíaca póstero-superior, desde donde se ubica un punto $1 \mathrm{~cm}$ más medial y $1 \mathrm{~cm}$ hacia caudal. Desde ese punto, se inserta el introductor en un ángulo de 45 grados a cefálico, apuntando hacia la apófisis espinosa de L5 (Figura 4).

\section{Agujas}

Fundamentalmente, las agujas espinales actualmente en uso entran en dos categorías principales: aquellas que cortan las fibras de la dura y las que las separan, sin cortarlas[4].

De las primeras, la más comúnmente usada es la aguja Quincke-Babcock (Figura 4). De las segundas, la Whitacre y la Sprotte son las más frecuentes (Figura 4). Una de las razones más importantes para la evolución hacia agujas espinales de menor diámetro y de punta no cortante la constituye la disminución

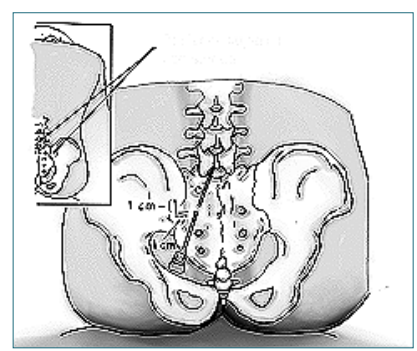

Figura 4. Abordaje de Taylor. Los puntos de reparo lo constituyen la espina ilíaca póstero-superior, desde donde se ubica un punto $1 \mathrm{~cm}$ más medial y $1 \mathrm{~cm}$ hacia caudal. Se inserta el introductor en un ángulo de 45 grados a cefálico, apuntando hacia la apófisis espinosa de L5. 
del riesgo de cefalea pospunción dural (CPPD), que se ha relacionado directamente al diámetro de las agujas empleadas y las características de su punta[5]. Si bien el concepto de ruptura de fibras meníngeas de acuerdo al diseño de la punta de la aguja y su diámetro es algo que se ha mantenido en el tiempo, estudios basados en modelos anatómicos de cadáveres con la posterior evaluación por medio de microfotografía electrónica, demuestran que las agujas con punta biselada generan un corte bastante neto de la duramadre, mientras que aquellas de punta cónica, dejan un orificio anfractuoso y mal delimitado. Lo más probable es que el defecto dejado por esta última generaría una respuesta inflamatoria más intensa que permitiría que el defecto meníngeo, principalmente de la duramadre, se cierre rápido y así evite el desarrollo del temido cuadro de cefalea[6] (Figura 5).

El otro factor involucrado es el número de punciones. Dado que el uso de agujas de menor diámetro potencialmente se asocia a mayor dificultad técnica, especialmente en estadios iniciales de entrenamiento, esto podría derivar en un mayor número de intentos, lo que atentaría contra el beneficio del uso de agujas más finas.

\section{Determinantes de la duración y extensión}

Es importante mencionar la definición de los conceptos de densidad, gravedad específica y baricidad de los anestésicos locales. Densidad es la relación entre la masa de una sustancia con respecto a su volumen. Esta relación varía con la temperatura, por lo que esta variable debe ser explicitada. La gravedad específica es la relación entre la densidad de la sustancia con respecto a un estándar como, por ejemplo un anestésico local a $20^{\circ} \mathrm{C}$ y el agua a $4{ }^{\circ} \mathrm{C}$. Finalmente, la baricidad, en forma análoga a la gravedad específica, es la relación entre la densidad de un anestésico local con respecto a la densidad de líquido cefalorraquídeo, ambos a $37^{\circ} \mathrm{C}$.

El uso de anestésicos locales en soluciones con densidades mayores o menores al del líquido cefalorraquídeo permite que pueda difundir dentro del espacio subaracnoideo a favor de la gravedad hacia porciones dependientes (drogas pesadas o hiperbáricas) o contra gravedad hacia segmentos no dependientes (drogas livianas o hipobáricas).

\section{Variaciones de la técnica tradicional}

\section{Anestesia sectorizada}

En la práctica, se posiciona a un paciente en decúbito lateral mientras se administra el anestésico local. Finalizada la inyección, el sujeto conserva la posición durante el tiempo necesario para la fijación de la droga, permitiendo a la droga distribuirse en forma no homogénea y unilateral. Consecuentemente, la anestesia clínica lograda es lateralizada, aunque la unilateralidad completa no se obtiene en todos los pacientes (sólo 50\%-70\% de los pacientes consiguen bloqueo sensitivo unilateral)[7]. Para favorecer este resultado, parece ser necesario utilizar bajas dosis de droga y agujas direccionadas hacia la zona de bloqueo. Más controvertido es la importancia de una velocidad de inyección lenta $\left(0,5 \mathrm{ml} \bullet \mathrm{min}^{-1}\right)$ con investigaciones clínicas a favor y en contra[8],[9].

Otra forma de anestesia espinal sectorizada es la técnica espinal en silla de montar o saddle block. En ésta es posible bloquear selectivamente las raicillas sacras, empleando anestésicos hiperbáricos, mientras el paciente se mantiene en posición sentada durante 5 minutos. Su nombre está dado porque el bloqueo sensitivo es casi exclusivamente del periné (S2-S4), en una distribución similar a lo que queda en contacto al sentarse en una silla de montar. En forma más extrema se ha publicado la anestesia espinal perianal selectiva[10]. Técnicamente se realiza igual al saddle block, pero usando $25 \%$ de la masa de la droga, consiguiendo un bloqueo de $\mathrm{S4}$.

Las ventajas de estas técnicas sectorizadas son

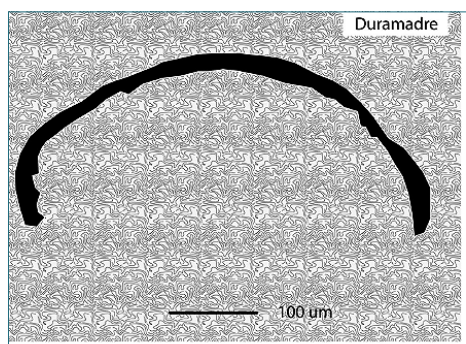

Aguja punta biselada

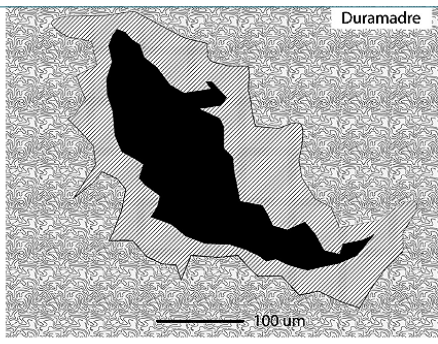

Aguja punta cónica
Figura 5. Microfotografía electrónica de muestra cadavérica. Comparación de la perforación de la dura hecha con agujas con punta biselada y con punta cónica. 
menor bloqueo simpático con menor repercusión hemodinámica y un perfil de recuperación que permite un alta precoz en caso de cirugía ambulatoria, además de una mejor aceptación por los pacientes[5].

\section{Anestesia espinal continua (CSA)}

Desde el comienzo de la historia de la anestesia espinal, la corta duración del efecto anestésico de la cocaína y procaína posteriormente, significó una limitación de la técnica. Los primeros intentos de prolongar el efecto se basaron en mantener agujas espinales in situ, para realizar inyecciones repetidas[11]. La primera descripción del uso de un catéter intratecal fue utilizando un catéter ureteral a través de una aguja espinal 14G[10]. El desarrollo de agujas y catéteres de menor diámetro dio impulso a la técnica hasta la aparición de microcatéteres (diámetro menor a 24G). La publicación de cuatro casos de síndrome de cauda equina, asociado al uso de microcatéteres espinales[12] y otros problemas técnicos de éstos, llevó a la prohibición de estos en Estados Unidos por la Administración de Alimentos y Drogas (Food and Drug Administration, FDA) en 1992. En ese reporte se sugiere que la causa del daño neurológico sería una mala distribución del anestésico local, asociado al uso de dosis elevadas de ellos. La prohibición de uso de microcatéteres significó la casi desaparición de la técnica espinal continua. El desarrollo de nuevos equipos ha permitido a la anestesia espinal continua resurgir siendo usada principalmente en cirugías de extremidades inferiores e intraabdominales en paciente añosos y con patologías concomitantes[13].

Actualmente, los equipos en uso son los micro catéteres insertados a través de una aguja espinal fina de 256 y el catéter sobre la aguja. En la técnica catéter sobre aguja, en primer lugar, se localiza el espacio peridural con un trocar biselado 18G. Por el interior de éste se desliza una aguja espinal con punta biselada 27G, que lleva montado un catéter 22G. Al atravesar la duramadre se observa salida de LCR por el extremo proximal del catéter, se desliza sobre la aguja 1-2 cm retirando posteriormente la aguja. Los resultados de ventajas y desventajas de uno $u$ otro son controversiales[14].

Una vez instalado el catéter, se administran pequeñas dosis de anestésicos, que se titulan hasta alcanzar el nivel requerido. Alcanzada una dosis que se ha estimado suficiente y no habiendo obtenido anestesia clínica adecuada se ha sugerido cambiar de baricidad, cambiar de anestésico local o abandonar la técnica por riesgo a que se esté frente a una situación de mala dis- tribución y riesgo de daño neurológico. Recomendaciones de buena práctica clínica incluyen el no sobrepasar dosis normales de inyección única y si no hay evidencia de bloqueo alguno, no insistir con la técnica[15].

Las ventajas frente a una técnica de inyección única son un menor riesgo de efectos hemodinámicos y respiratorios adversos y el tener un catéter que puede prolongar la anestesia o la analgesia postoperatoria. Las desventajas son una mayor tasa de fallas[15], riesgo de aparición de cefalea entre 1 y 10\%[16] y un teórico mayor riesgo de infección por dejar un cuerpo extraño. La posibilidad de daño neurológico es difícil de establecer actualmente. Las series clínicas publicadas han reportado un bajo número de pacientes expuestos a la técnica, aunque aparentemente no serían mayores que la de inyección única.

Al comparar la CSA con la técnica combinada espinal epidural (CEE), los resultados de estudios clínicos muestran ventajas y desventajas para ambos, siendo difícil demostrar superioridad absoluta de uno sobre otro. La CEE es probablemente más popular actualmente frente a la mayor incidencia de cefalea con CSA, la aún reciente historia de lesión de cauda equina y los temores a equivocaciones por personal no entrenado. Además, el concepto que el espacio intratecal es más vulnerable que el peridural es otro posible factor del menor uso de la técnica en la actualidad frente a CEE.

\section{Anestesia combinada espinal epidural (CEE)}

La anestesia combinada espinal epidural combina las ventajas de las técnicas espinal y peridural, disminuyendo las desventajas. Esencialmente, conseguimos con el componente intratecal una menor latencia de acción, mayor eficacia en el bloqueo, con una baja dosis de anestésicos y el consiguiente menor riesgo de toxicidad sistémica. Por su parte, dejar un catéter en el espacio peridural permite titular la dosis y evitar niveles de bloqueo extenso, prolongar la anestesia en caso de cirugía de larga duración y mantener analgesia neuraxial en el período posoperatorio.

Técnicamente, las primeras descripciones de la CEE son de 2 punciones secuenciales: inyección intratecal seguida de una segunda punción peridural en otro ínterespacio. Sin embargo, la técnica de aguja sobre aguja popularizó la CEE al simplificar los pasos[16]. En ésta, en primer lugar (previa marcación del nivel adecuado y asepsia correspondiente) se realiza la punción con la aguja peridural. Una vez identificado el espacio peridural se procede a introducir por dentro de la aguja peridural una aguja espinal de un largo superior al peridural, hasta puncionar la duramadre-aracnoides 
y obtener reflujo de LCR. Se inyecta el anestésico, se retira la aguja espinal y a continuación se introduce el catéter en el espacio peridural. Las variaciones actuales tienen que ver con diferentes equipos introducidos por los distintos fabricantes. La mayoría de las modificaciones introducidas a las agujas se relacionan con la disminución del riesgo potencial de cateterización intratecal y de evitar el desplazamiento de la aguja espinal dentro de la aguja peridural debido a la discordancia de tamaño, lo cual podría favorecer en teoría, una falla en la inyección intratecal, por un movimiento inadvertido de la aguja espinal.

Existen varias alternativas en el manejo clínico de la CEE[17]. La primera es usar el componente espinal con dosis completa tal cual una técnica de inyección única y posteriormente, durante el periodo de regresión de ésta, iniciar el uso del catéter peridural. También es posible usar una baja dosis por el componente espinal y suplementarlo vía peridural para lograr un ajuste más fino del nivel a una velocidad más controlada, evitando los efectos adversos de un bloqueo simpático exagerado y de rápida instalación.

La interacción entre ambos componentes está sujeta a varias teorías: En primer lugar, la simple inyección de un volumen de solución en el espacio peridural induce aumento de la presión en el espacio peridural, lo que deforma y comprime el saco dural de tal manera que se favorece una mayor dispersión del anestésico intratecal y aumento de la altura de bloqueo alcanzado. Esto es la técnica EVE o extensión por volumen epidural [18]. Clínicamente se ha demostrado que la inyección de solución salina antes de 20 minutos de la inyección intratecal produce aumento en al menos dos niveles del bloqueo máximo alcanzado. En segundo lugar, la sinergia que se produce al bloquear las fibras nerviosas en puntos distintos como el recorrido intratecal y el recorrido por el espacio peridural y ganglio sensitivo dorsal facilita el bloqueo nervioso, a pesar de no lograr un bloqueo sólido de los segmentos por separado. En tercer y último lugar, también se ha postulado la posibilidad de paso de anestésico desde el espacio peridural a través de la duramadre-aracnoides, por el orificio producido por la aguja espinal.

La maniobra de extensión por volumen epidural puede disminuir la duración del bloqueo sensitivo si se realiza tardíamente. Un posible beneficio agregado es una regresión más rápida del bloqueo motor ( 60 min) comparado con una dosis intratecal única, en pacientes sometidas a operación cesárea, lo que pudiera tener un impacto positivo en la disminución del tiempo de estadía en una unidad de recuperación postoperatoria. Finalmente, el efecto compresor del saco dural por la maniobra EVE, persiste por al menos 30 minutos[18].
Las controversias más habituales de esta técnica son si existe mayor riesgo de cateterización intratecal, el por qué su tasa de falla del componente espinal, la incidencia de cefalea, la probabilidad de daño neurológico, la posibilidad y consecuencia de la inyección de partículas metálicas en el espacio subaracnoideo e infecciones.

La presencia de un orificio dural, por aguja espinal fina, en un lugar próximo a la introducción de un catéter hace suponer la posibilidad de que se favorezca la introducción de éste en el espacio subaracnoideo. En un trabajo en cadáveres se demostró la dificultad e improbabilidad que esto suceda[19]. A pesar de esto las compañías fabricantes de equipos han desarrollado agujas que hagan aún menos probable la cateterización otorgándole al componente espinal y al catéter epidural orientaciones distintas (Figura 6).

Aunque existen trabajos que demuestran daños microscópicos en las puntas de las agujas espinales finas, luego de ser introducidas por el interior de las epidurales usadas durante la técnica CEE, no hay evidencia clínica de daño por este motivo.

Algunas series muestran fallas en la eficacia del componente intratecal con una frecuencia mayor a la esperada en una anestesia espinal por punción única. Varias explicaciones se han sugerido: en primer lugar, la identificación del espacio peridural en un ángulo de abordaje oblicuo que lleva al componente espinal errando tangencialmente el saco dural; en segundo lugar, la falta de fuerza en el eje de la aguja haciéndola incapaz de atravesar el saco dural "rebotando" contra éste; en tercer lugar, una longitud insuficiente de la aguja espinal para sobresalir de la aguja epidural; y por último, por deslizamiento de la aguja espinal dentro de la aguja peridural, retrocediendo y saliendo del saco dural e inyectando fuera del espacio subaracnoideo.

La incidencia de complicaciones como la cefalea pospunción dural no parece ser mayor que la de ambas técnicas por separado. Tampoco la incidencia de lesiones neurológicas a pesar del temor de que la

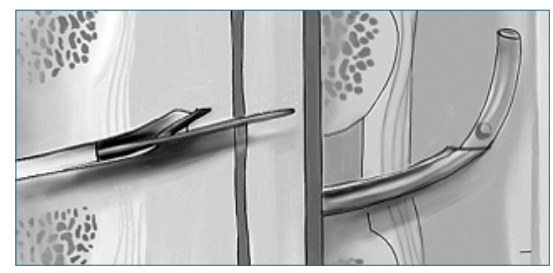

Figura 6. Diseño de aguja para anestesia combinada espinal-epidural. El componente espinal y la salida del catéter epidural tienen orientaciones distintas. 
técnica podría favorecerlas. Al introducir el catéter peridural, luego de la inyección espinal, el efecto anestésico en curso podría impedir que el paciente refiriese parestesias. Sin embargo, con respecto a este punto, la incidencia de parestesias reportadas es semejante que la técnica peridural normal.

\section{Referencias}

1. Hogan QH. Tuffier's line: the normal distribution of anatomic parameters. Anesth Analg. 1994 Jan;78(1):194-5. https:// doi.org/10.1213/00000539199401000-00044 PMID:8267168

2. Bromage $P R$, van Zundert $A$, van Steenberge A, Corke BC, Fragraeus L. A loss-of-resistanceto-negative-pressure test for subarachnoid puncture with narrow-gauge needles. Reg Anesth. 1993 May-Jun;18(3):155-61. PMID:8323887

3. Taylor J. Lumbosacral subarachnoid tap. J Urol. 1940;43(4):5614. https://doi.org/10.1016/ S0022-5347(17)71435-8.

4. Tsen LC, Hepner DL. Needles used for spinal anesthesia. Expert Rev Med Devices. 2006 Jul;3(4):499-508. https://doi. org/10.1586/17434440.3.4.499 PMID:16866646

5. Ready LB, Cuplin S, Haschke $\mathrm{RH}$, Nessly M. Spinal needle determinants of rate of transdural fluid leak. Anesth Analg. 1989 Oct;69(4):457-60. https:// doi.org/10.1213/00000539198910000-00006 PMID:2782645

6. Reina MA, de Leon-Casasola OA, López A, De Andres J, Martin S, Mora M. An in vitro study of dural lesions produced by 25-gauge Quincke and Whitacre needles evaluated by scanning electron microscopy. Reg Anesth Pain Med. 2000 JulAug;25(4):393-402. https://doi. org/10.1053/rapm.2000.7622 PMID:10925937

7. Casati A, Fanelli G. Unilateral spinal anesthesia. State of the art. Minerva Anestesiol. 2001 Dec;67(12):855-62.

PMID:11815746

8. Enk D, Prien $\mathrm{T}$, Van Aken $\mathrm{H}$, Mertes N, Meyer J, Brüssel

$T$. Success rate of unilateral spinal anesthesia is dependent on injection flow. Reg Anesth Pain Med. 2001 SepOct;26(5):420-7. https://doi. org/10.1053/rapm.2001.26489 PMID:11561261

9. Casati A, Fanelli G, Cappelleri G, Aldegheri $G$, Berti M, Senatore $R$, et al. Effects of speed of intrathecal injection on unilateral spinal block by $1 \%$ hyperbaric bupivacaine. A randomized, double-blind study. Minerva Anestesiol. 1999 Jan-Feb;65(1-2):5-10. PMID:10206032

10. Wassef MR, Michaels El, Rangel JM, Tsyrlin AT. Spinal perianal block: a prospective, randomized, double-blind comparison with spinal saddle block. Anesth Analg. 2007 Jun;104(6):1594-6. https://doi.org/10.1213/01. ane.0000261510.37489.00 PMID:17513664

11. Tobias G, Sands RP Jr, Bacon DR. Continuous spinal anesthesia: a continuous history? Reg Anesth Pain Med. 1999 Sep-Oct;24(5):453-7. PMID:10499758

12. Rigler ML, Drasner $K$, Krejcie TC, Yelich SJ, Scholnick FT, DeFontes J, et al. Cauda equina syndrome after continuous spinal anesthesia. Anesth Analg. 1991 Mar;72(3):275-81. https:// doi.org/10.1213/00000539199103000-00001 PMID:1994754

13. Bevacqua BK. Continuous spinal anaesthesia: what's new and what's not. Best
Pract Res Clin Anaesthesiol. 2003 Sep;17(3):393-406. https://doi.org/10.1016/ S1521-6896(02)00117-9 PMID: 14529010

14. De Andrés J, Valía JC, Olivares A, Bellver J. Continuous spinal anesthesia: a comparative study of standard microcatheter and Spinocath. Reg Anesth Pain Med. 1999 Mar-Apr;24(2):110-6. https:// doi.org/10.1097/00115550199924020-00003 PMID:10204895

15. De Andrés JA, Febré E, Bellver J, Bolinches R. Continuous spinal anaesthesia versus single dosing. A comparative study. Eur J Anaesthesiol. 1995 Mar;12(2):135-40. PMID:7781632

16. Denny NM, Selander DE. Continuous spinal anaesthesia. $\mathrm{Br} J$ Anaesth. 1998 Oct;81(4):590-7. https://doi.org/10.1093/ bja/81.4.590 PMID:9924237

17. Cook TM. Combined spinalepidural techniques. Anaesthesia. 2000 Jan;55(1):42-64. https://doi.org/10.1046/j.13652044.2000.01157.xPMID:10594432

18. McNaught AF, Stocks GM. Epidural volume extension and low-dose sequential combined spinal-epidural blockade: two ways to reduce spinal dose requirement for caesarean section. Int J Obstet Anesth. 2007 Oct;16(4):346-53. https://doi. org/10.1016/j.ijoa.2007.03.013 PMID:17693082

19. Holmström $B$, Rawal N, Axelsson K, Nydahl PA. Risk of catheter migration during combined spinal epidural block: percutaneous epiduroscopy study. Anesth Analg. 1995 Apr;80(4):747-53. PMID:7893029 